\title{
AVALIAÇÃO DA ESTABILIDADE DE SOLUÇÕES MODELO (CMC-SACAROSE) EM RECONGELAMENTOS
}

\author{
DOUGLAS FERNANDES BARBIN* \\ LENA SOLEDAD MONZON DAVILA** \\ VIVALDO SILVEIRA JUNIOR ${ }^{\star \star \star}$
}

\begin{abstract}
O objetivo deste trabalho foi avaliar a estabilidade de soluções modelo pela determinação da temperatura de início de congelamento depois de repetidos processos de congelamento. As soluções utilizadas eram compostas por água, sacarose e Carboxi-Metil-Celulose (CMC). Foram avaliadas as concentrações de sacarose de 15 e $31,1 \%(\mathrm{~m} / \mathrm{m}$ total da amostra) e do espessante de 0,5, 1 e 1,5\% ( $\mathrm{m} / \mathrm{m}$ total da amostra). Buscou-se determinar a possibilidade de reutilização de soluções modelo em ensaios de congelamento. Com os resultados obtidos pode-se concluir que para a concentração de espessante de $0,5 \%$ não houve alteração da capacidade de retenção de água. A variação da concentração de sacarose não influenciou a estabilidade das soluções durante o estudo.
\end{abstract}

PALAVRAS-CHAVE: CONGELAMENTO; PROPRIEDADES TERMOFÍSICAS; TRANSFERÊNCIA DE CALOR.

* Mestre em Engenharia de Alimentos, Laboratorio de Automacao e Controle de Processos em Alimentos (LACPA), Departamento de Engenharia de Alimentos, Faculdade de Engenharia de Alimentos (FEA) Universidade Estadual de Campinas (UNICAMP), Campinas, SP (e-mail:dfbarbin@yahoo.com.br).

** Mestre em Engenharia de Alimentos, LACPA, Departamento de Engenharia de Alimentos, FEA, UNICAMP, Campinas, SP (e-mail: lena@fea.unicamp.br).

*** Professor, Doutor em Engenharia de Alimentos, LACPA, Departamento de Engenharia de Alimentos, FEA, UNICAMP, Campinas, SP (e-mail: vivaldo@fea.unicamp.br). 


\section{INTRODUÇÃO}

As propriedades térmicas dos alimentos incluem a condutividade térmica, difusividade e calor específico, entre outras. Em processos de congelamento, tais propriedades são essenciais para a simulação da variação de temperatura no interior dos alimentos e estimativas de tempo de congelamento e carga térmica do produto (SAAD e SCOTT, 1996; BECKER e FRICKE, 1999; RENAUD et al. 1992; RESENDE e SILVEIRA JR., 2002a).

Alternativa comum em estudos de propriedades térmicas de alimentos envolve a utilização de soluções modelo para representar os métodos experimentais. Diversos autores utilizaram soluções modelo no estudo das propriedades dos alimentos, ou como simulação de produtos alimentícios. Segundo BECKER e FRICKE (1999), as propriedades termofísicas dos alimentos são muito difíceis de se estimar devido à grande variação da sua composição e temperatura. Uma alternativa é estimar as propriedades termofísicas dos alimentos utilizando soluções modelo, cuja composição tem propriedades termofísicas conhecidas, eliminando assim os efeitos da composição e da temperatura. Alguns dados de composições podem ser encontrados em diversas referências (HOLLAND et al., 1991; USDA, 2008).

RENAUD et. al. (1992), PONGSAWATMANIT e MIYAWAKI (1993), utilizaram soluções binárias para avaliar a dependência da fração de gelo da temperatura de congelamento. RESENDE, NEVES FILHO e SILVEIRA (2002) e RESENDE e SILVEIRA JR. (2002a, b) empregaram soluções modelo compostas de $10 \%$ de sacarose e $0,5 \%$ de goma "k-carragenan" para a simulação de polpas de frutas durante o processo de congelamento. BERTO et al. (2003) utilizaram solução com $65 \%$ de sacarose e $0,1 \%$ de goma carboximetilcelulose (CMC) para a simulação de suco de frutas submetido ao processo de tratamento térmico.

Durante os processos de congelamento dos alimentos, a transferência de calor depende das frações mássicas e volumétricas de cada componente do produto. As frações de gelo e de água líquida nos alimentos, durante o processo de congelamento, são dependentes da temperatura (FIKIIN e FIKIIN, 1999).

O ponto inicial de congelamento constitui importante propriedade termofisica nos processos de congelamento, não apenas para determinação das melhores condições de estocagem, mas também para a avaliação das alterações provocadas pelo processo no produto.

A temperatura de início de congelamento de alimentos ou soluções é o indicador da proporção da quantidade de água ligada. Essa quantidade pode demonstrar o grau de irreversibilidade das ligações da água após o descongelamento.

Segundo os trabalhos de BECKER e FRICKE (1999), SAAD e SCOTT (1996), o ponto inicial de congelamento é mais baixo que o ponto de congelamento da água pura devido às substâncias presentes no alimento. Nesse instante, uma parte da água cristaliza e o restante forma solução mais concentrada. Esse fenômeno provoca variação nas suas propriedades devido ao aumento da concentração de solutos com a formação dos cristais de gelo (SCOTT, BECK e HELDMAN, 1992; SAAD e SCOTT, 1996).

O presente trabalho teve como o objetivo geral avaliar a estabilidade de soluções modelo pela determinação da temperatura de início de congelamento depois de repetidos processos de congelamento. O objetivo específico foi determinar a possibilidade de reutilização de soluções modelo em ensaios de congelamento.

\section{MATERIAL E MÉTODOS}

\subsection{PREPARAÇÃO DAS AMOSTRAS}

Foram preparadas duplicatas de amostras de $100 \mathrm{~g}$ da solução modelo, compostas por água, sacarose e Carboxi-Metil-Celulose (CMC). 
Os processos de congelamento em alimentos variam de acordo com a composição básica do produto (SAAD e SCOTT, 1996). Os testes foram planejados de forma a se verificar a influência da concentração de sacarose e do espessante (CMC) na solução. Para avaliação dos efeitos do espessante na estabilidade dos recongelamentos foram preparadas soluções com 0,5\%, 1,0\% e 1,5\% ( $\mathrm{m} / \mathrm{m}$ total da amostra) de CMC e $30 \%$ ( $\mathrm{m} / \mathrm{m}$ total da amostra) de sacarose. Para avaliação do efeito da sacarose foram usadas soluções de $15 \%$ e $31,1 \%$ ( $\mathrm{m} / \mathrm{m}$ total da amostra) com $0,5 \%$ de CMC ( $\mathrm{m} / \mathrm{m}$ total da amostra).

As amostras foram acondicionadas em embalagens de polietileno e colocadas em freezer doméstico para determinação do perfil de temperatura durante o período de congelamento. A partir do perfil de temperatura determinou-se a temperatura inicial de congelamento das amostras.

Usou-se freezer doméstico por permitir a avaliação da cinética de congelamento das amostras devido à baixa velocidade do congelamento, possibilitando a determinação da temperatura inicial de congelamento (JEREMIAH, 1996).

\subsection{PERFIS DE TEMPERATURA PONTO DE INÍCIO DE CONGELAMENTO}

De acordo com diversos autores, os métodos mais usados para a determinação de temperatura de início de congelamento são baseados em dados experimentais de tempo versus temperatura, entalpia versus temperatura ou de osmometria crioscópica. Dentre esses métodos, o mais comumente usado devido à praticidade é o registro gráfico dos dados experimentais de temperatura em função do tempo durante o congelamento (FENNEMA, POWRI e MARTH, 1973).

As temperaturas das amostras durante o processo de congelamento foram monitoradas por termopares tipo $\mathrm{T}$ (cobre-constantan). Os sinais elétricos foram enviados para equipamento de aquisição de dados (Lynx Tecnologia Eletrônica Ltda., SP, BR, modelo CAD 12/32), responsável pela leitura. A aquisição mantinha conexão remota com o microcomputador, que armazenou os dados de temperatura.

Para todos os ensaios realizados, o ponto inicial de congelamento foi determinado a partir das curvas de temperatura obtidas durante o processo de congelamento das amostras.

A relação tempo-temperatura de produtos submetidos ao congelamento pode ser esquematizada conforme a Figura 1, que ilustra a variação de temperatura da água pura (ASB) e de determinado alimento (AS'B'C') com o decorrer do tempo de exposição a um sistema de congelamento.

A primeira parte do gráfico (trecho AS ou AS') mostra a etapa de redução da temperatura inicial do produto mediante retirada do calor sensível (em que ainda não ocorre mudança de fase) até o sub-resfriamento abaixo do ponto de solidificação do alimento. Esse ponto, diferentemente da água, está sempre abaixo de $0^{\circ} \mathrm{C}$ devido à presença de sólidos dissolvidos no produto e antes do início da formação dos cristais de gelo. A água presente no alimento no ponto $S$ ainda se encontra no estado líquido, apesar de estar abaixo do seu ponto de congelamento. Esse fenômeno, conhecido como super-resfriamento pode chegar a até $10^{\circ} \mathrm{C}$ abaixo do ponto de congelamento do alimento e varia de acordo com seu tipo de composição e taxa de resfriamento (FELLOWS, 2000; ZARITZKI, 2000). Os pontos S e S' representam o início da nucleação, quando os cristais de gelo começam a liberar o calor latente de solidificação mais rapidamente do que a perda de calor para o meio. Isto causa o aumento da temperatura até B ou B', que representa o ponto inicial de congelamento. Os alimentos apresentam temperatura menor que a água pura nesse ponto, devido à presença de sólidos solúveis. Além disso, o trecho a partir de $\mathrm{B}$ representa o período em que ocorre o aumento dos cristais de gelo e a remoção do calor latente. A parte parcialmente congelada não reduz a temperatura até que toda a água disponível esteja congelada, apesar do calor ser removido nas mesmas condições. Esse patamar de temperatura (trecho $B^{\prime} C^{\prime}$ ) não é constante nos alimentos, uma vez que ocorre o aumento da concentração dos sólidos da solução durante o 
congelamento, reduzindo sutil e continuamente o ponto de congelamento da água que continua disponível. Após o congelamento, a temperatura continua a diminuir com a retirada do calor sensível abaixo do ponto de congelamento (após $C^{\prime}$ ) até que a temperatura do alimento se aproxime do valor da temperatura do meio de resfriamento (ZARITZKI, 2000). O mesmo processo foi descrito por FELLOWS (2000) com a seguinte observação: existe pequena variação nos alimentos durante o processo de remoção do calor latente, em que os solutos tornam-se supersaturados e cristalizam. O calor latente de cristalização dos solutos é liberado e a temperatura aumenta até atingir o valor da temperatura eutética para o soluto, isto é, quando o soluto está em equilíbrio com a solução não congelada. Segundo o mesmo autor, após esse processo, continua ocorrendo a cristalização da água e dos solutos até o final da etapa de retirada do calor latente do produto. Esse fenômeno está representado pelo trecho C-D na Figura 1.

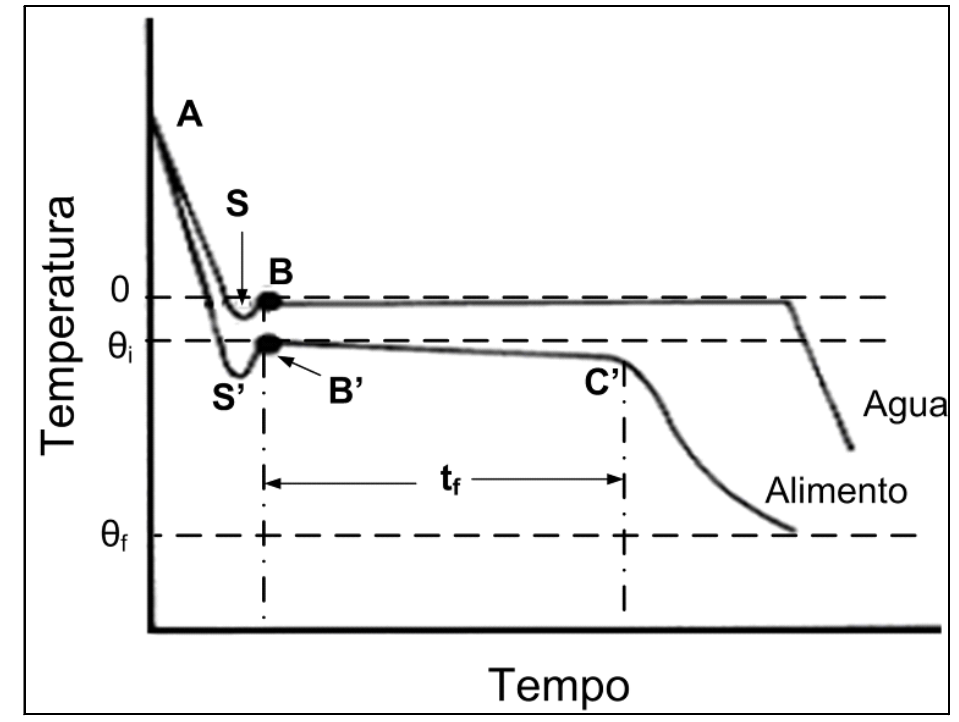

\section{FIGURA 1 - VARIAÇÃO DA TEMPERATURA DURANTE O PROCESSO DE CONGELAMENTO DE ALIMENTOS}

(Fonte: ZARITZKI, 2000).

Neste trabalho, as amostras foram resfriadas a partir da temperatura ambiente até $-20^{\circ} \mathrm{C}$, e em seguida descongeladas. Efetuou-se o processo de congelamento e descongelamento por seis vezes para cada solução. Esses ensaios foram realizados em triplicatas para se avaliar a estabilidade térmica das soluções modelo pela determinação dos pontos iniciais de congelamento, mostrando as alterações provocadas por repetidos processos de congelamento e descongelamento.

\section{RESULTADOS E DISCUSSÃO}

As curvas de temperatura do produto durante o congelamento mostraram que após o subresfriamento ocorreu aumento na temperatura, decorrente da formação dos primeiros cristais de gelo e da liberação de calor latente. Logo, houve a formação de "pseudopatamar", conforme descrito na literatura, quando a maior parte da água era convertida em gelo. A temperatura de início de congelamento foi determinada no momento em que ocorreu o início dessa fase, seguida por abaixamento rápido da temperatura como exemplificado na Figura 1. 
Os resultados de temperatura inicial de congelamento para as amostras com 0,5\%, 1,0\% e $1,5 \%$ de CMC e $15 \%$ e 30\% de sacarose são apresentados nas Figuras 2, 3 e 4.

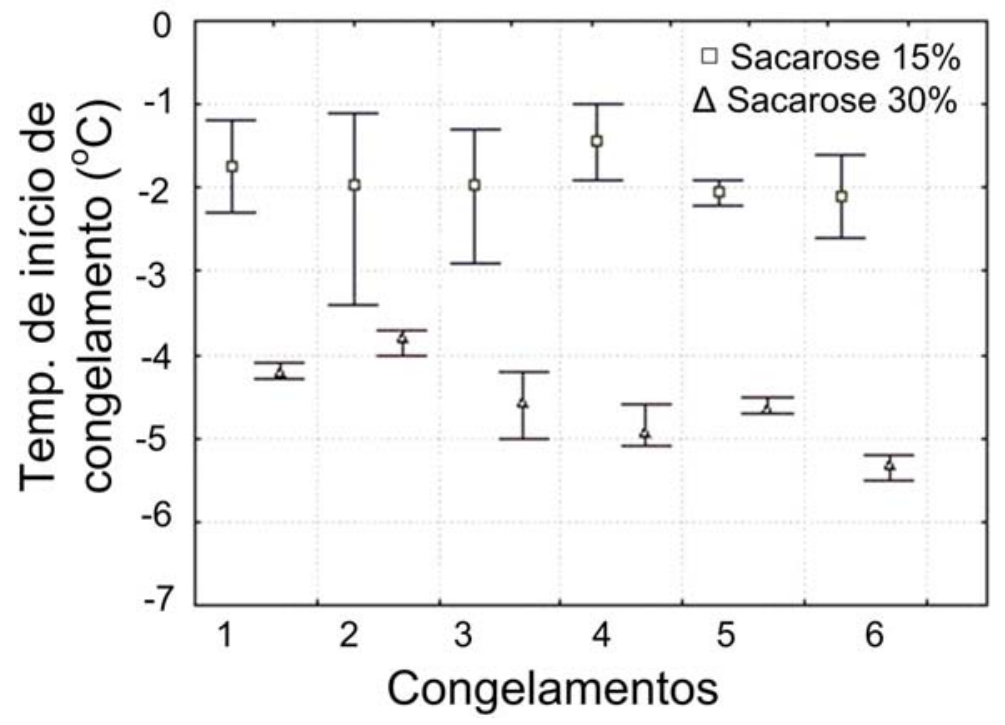

FIGURA 2 - VARIAÇÃO DA TEMPERATURA INICIAL DE CONGELAMENTO OBTIDA PARA VÁRIOS RECONGELAMENTOS DAS AMOSTRAS DE SOLUÇÃO MODELO COM DIFERENTES CONCENTRAÇÕES DE SACAROSE E 0,5\% DE ESPESSANTE (CMC)

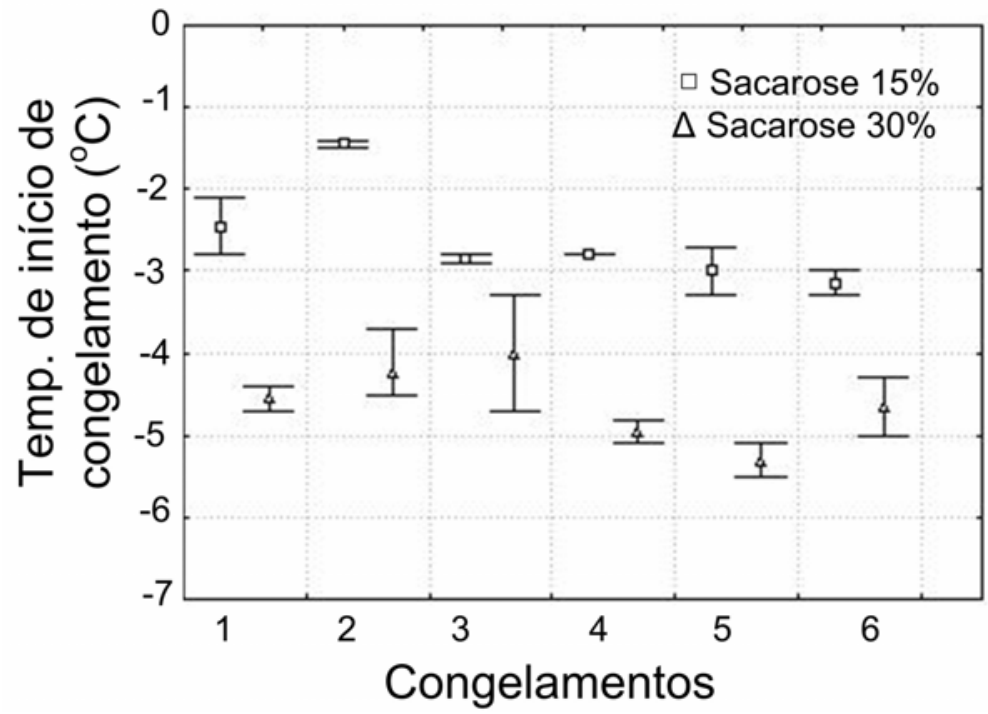

FIGURA 3 - VARIAÇÃO DA TEMPERATURA INICIAL DE CONGELAMENTO OBTIDA PARA VÁRIOS RECONGELAMENTOS DAS AMOSTRAS DE SOLUÇÃO MODELO COM DIFERENTES CONCENTRAÇÕES DE SACAROSE E 1,0\% DE ESPESSANTE (CMC)

Os resultados obtidos (Figuras 2, 3 e 4) indicaram que o ponto de início de congelamento apresenta valores praticamente constantes para cada uma das amostras analisadas após seis congelamentos. Demonstram também que não existe tendência de alteração do ponto inicial de congelamento com a repetição dos processos de congelamento e recongelamento para as amostras analisadas. 
RESENDE e SILVEIRA JR. (2002a, b) e RESENDE, NEVES FILHO e SILVEIRA JR. (2002) determinaram experimentalmente algumas propriedades térmicas de soluções modelo compostas por $10 \%$ de sacarose e $0,5 \%$ de goma "k-carragenan". Os resultados mostraram diferenças variando de $6 \%$ a $33 \%$ nos valores de acordo com o método utilizado. As amostras foram posteriormente utilizadas na simulação de polpas de frutas durante o processo de congelamento em diversas condições.

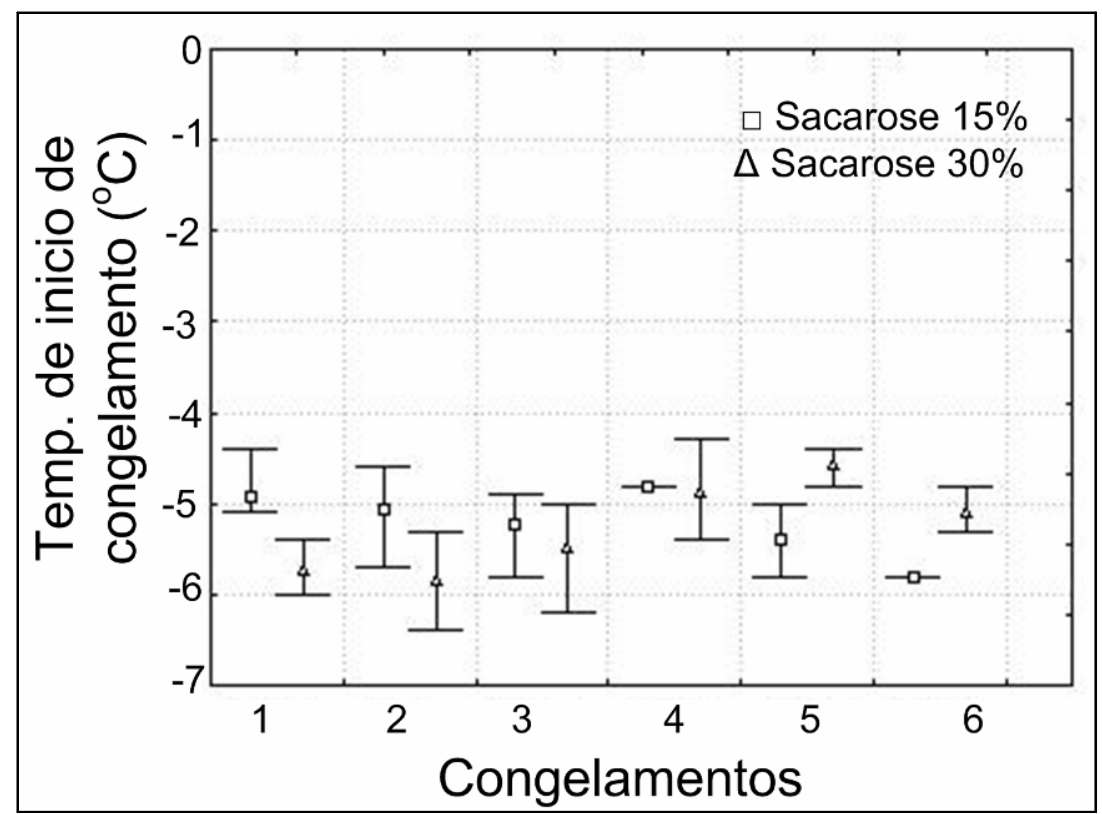

\section{FIGURA 4 - VARIAÇÃO DA TEMPERATURA INICIAL DE CONGELAMENTO OBTIDA PARA VÁRIOS RECONGELAMENTOS DAS AMOSTRAS DE SOLUÇÃO MODELO COM DIFERENTES CONCENTRAÇÕES DE SACAROSE E 1,5\% DE ESPESSANTE (CMC)}

A estabilidade térmica da solução com $65 \%$ de sacarose e $0,1 \%$ de goma carboximetilcelulose, tratada a $60^{\circ} \mathrm{C}$ durante 4 horas, foi avaliada por BERTO et al. (2003). Os resultados indicaram que a solução analisada não sofria alterações, possibilitando seu reprocessamento.

Os resultados obtidos para as amostras com diferentes concentrações de sacarose mostraram redução do ponto de congelamento com o aumento da concentração de sacarose, porém não apresentaram diferença significativa da temperatura inicial de congelamento após os recongelamentos.

\section{CONCLUSÃO}

A partir da análise dos resultados pode-se concluir que a variação da concentração de espessante $(0,5 \%, 1 \%$ e 1,5\%) para ambas as concentrações de sacarose ( $15 \%$ e $30 \%)$ não provocou alteração da temperatura de início de congelamento após repetidos processos. Esse fato evidencia a não ocorrência de rearranjo da estrutura de sólidos do alimento, indicando que não houve alteração da capacidade de retenção de água por parte do produto.

Os resultados impelem a novos estudos para avaliação da irreversibilidade da ligação da água na estrutura do espessante nos subsequentes recongelamentos, visando verificar se ocorre redução da retenção de água ligada. 
Devido à não significância dos resultados obtidos neste trabalho sobre alterações da temperatura inicial de congelamento nos processos de repetição do congelamento, conclui-se pela possibilidade de reutilização da solução nessas concentrações em estudos de congelamento.

\section{ABSTRACT}

\section{MODEL SOLUTIONS (CMC-SUCROSE) STABILITY EVALUATION AFTER SEVERAL FREEZING PROCESS}

The objective of this paper was to evaluate the stability of the model solutions determining initial freezing temperature after repeated freezing process. The solutions were composed by water, sucrose and Carboxymethylcellulose (CMC). Were evaluated the concentrations of sucrose from $15 \%$ to $31.1 \%(\mathrm{w} / \mathrm{w})$ and the concentration of $\mathrm{CMC}$ from $0.5 \%, 1.0 \%$ to $1.5 \%(\mathrm{w} / \mathrm{w})$. It was also studied the possibility of reutilization of the model solutions for freezing tests. The results showed that at the concentration of $0.5 \%$ there was no alteration at the water binding capacity. The variation of sucrose concentration did not interfered in the stability of the solution during the study

KEY-WORDS: FREEZING PROCESS; THERMO PHYSICAL PROPERTIES; HEAT TRANSFER.

\section{REFERÊNCIAS}

1 BECKER, B.R.; FRICKE, B.A. Food thermophysical property models. Int. Comm. Heat and Mass Transfer, v.26, n.5, p.627-636, 1999.

2 BERTO, M.I.; GRATÃO, A.C.A.; SILVEIRA JR.; V.; VITALI, A.A. Solução modelo de sacarose e CMC: análise do tempo de hidratação, caracterização reológica e estabilidade térmica. Brazilian J. Food Techn., v.6, n.107, p.9-14, 2003.

3 FELLOWS, P.J. Food processing technology: principles and practice. Cambridge, UK: Woodhead Publishing, 2000. 575 p.

4 FIKIIN, K.A.; FIKIIN, A.G. Predictive equations for thermophysical properties and enthalpy during cooling and freezing of food materials. J. Food Eng., v.40, p.1-6, 1999.

5 FENNEMA, O.R; POWRI, W.D.; MARTH, E.H. Low temperature preservation of foods and living matter. New York: Marcel Decker, 1973.

6 HOLLAND, B.; WELCH, A. A.; UNWIN, I. D.; BUSS, D. H.; PAUL, A. A.; SOUTHGATE, D. A. T. McCance \& Widdowson's: the composition of foods. $5^{\text {th }}$ ed. Cambridge, UK: Royal Society of Chemistry, 1991. 554 p.

7 JEREMIAH, L.E. Freezing effcts on food quality. New York: Marcel Dekker, 1996. 520 p.

8 PONGSAWATMANIT, R.; MIYAWAKI, O. Measurement of temperature-dependent ice fraction in frozen foods. Bioscience, Biotechnology and Biochemistry, v. 57, n. 10, p. 1650-1654, 1993.

9 RENAUD, T. et al. Thermal properties of model foods in the frozen state. J. Food Eng., v.15, p.83-97, 1992.

10 RESENDE, J.V.; NEVES FILHO, L.C.; SILVEIRA JR., V. Coeficientes de transferência de calor efetivos no congelamento com ar forçado de modelos de polpas de frutas em caixas comerciais. Brazilian J. Food Techn., v.5, p.33-42, 2002.

11 RESENDE, J.V.; SILVEIRA JR., V. Medidas da condutividade térmica efetiva em modelos de polpas de frutas no estado congelado. Ciência e Tecnologia de Alimentos, v.22, n.2, p.177-183, 2002a.

12 RESENDE, J.V.; SILVEIRA JR., V. Escoamento de ar através de embalagens de polpa de frutas em caixas comerciais: efeitos sobre os perfis de velocidade em túneis de congelamento. Ciência e Tecnologia de Alimentos, v.22, n.2, p.184-191, 2002b.

13 SAAD, Z.; SCOTT, E.P. Estimation of temperature dependent thermal properties of basic food solutions during freezing. J. Food Eng., v.28, p.1-19, 1996. 
14 SCOTT, E.P.; BECK, J.V.; HELDMAN, D.R. Estimation of time variable heat transfer coefficients in frozen foods during storage. J. Food Eng., v.15, p.99-121, 1992.

15 USDA. United States Department of Agriculture. Agriculture Research Service. USDA National Nutrient Database for Standard Reference. Composition of foods raw, processed, prepared. Release 21. Beltsville, Maryland, 2008. 49 p.

16 ZARITZKI, N.E. Factors affecting the stability of frozen foods. In: KENNEDY, C.J. Managing frozend foods. Cambridge: Woodhead Publishing, 2000. 